\title{
TTR
}

Traduction, terminologie, re?daction

\section{La traduction de la langue pure : fondation de la littérature}

\section{Catherine Mavrikakis}

Volume 2, numéro 1, 1er semestre 1989

Carrefours de la traduction

URI : https://id.erudit.org/iderudit/037033ar

DOI : https://doi.org/10.7202/037033ar

Aller au sommaire du numéro

Éditeur(s)

Association canadienne de traductologie

ISSN

0835-8443 (imprimé)

1708-2188 (numérique)

Découvrir la revue

Citer cet article

Mavrikakis, C. (1989). La traduction de la langue pure : fondation de la littérature. TTR, 2(1), 59-74. https://doi.org/10.7202/037033ar

Tous droits réservés (C) TTR: traduction, terminologie, rédaction — Les auteurs, d'utilisation que vous pouvez consulter en ligne.

https://apropos.erudit.org/fr/usagers/politique-dutilisation/ 


\section{La traduction de la langue pure: fondation de la littérature}

\section{Catherine Mavrikakis}

\section{La perte de la langue maternelle ou la langue fait l'épreuve de l'étranger}

«Seule demeure la langue maternelle» (Arendt, 1980, p. 19), lorsque la mémoire a accompli le cycle de son oubli. Tel serait le mythe que l'on pourrait désigner comme constitutif de notre pensée de la langue. La langue maternelle se verrait dotée des pouvoirs de l'objet-ruine: elle se dresse dans l'horizon humain lorsque tout a été effacé ou perdu. Pourtant la littérature s'inscrit souvent en faux contre cette théorie d'une éternelle plénitude de la langue. Voici qu'à lire les écrivains, les poètes, les philosophes ou encore les linguistes, on s'aperçoit vite que la langue maternelle n'est pas donnée à celui qui la parle, et que l'appropriation de ce qui apparaît au départ comme le plus familier ne va pas de soi. La langue maternelle alors n'est plus le sein que le petit homme pourra toujours venir téter, mais représente une matière, une production que l'homme doit faire sienne au fur et à mesure qu'il la crée. La langue maternelle perd son statut de mère nourricière et omniprésente et ne peut échapper à un travail de réappropriation. En ce sens, une réflexion sur la langue se réclame de l'héritage d'un Humboldt qui écrivait: «On doit voir le langage non tant comme une chose produite, morte, mais bien davantage comme une production. [Le langage] lui-même n'est pas une đuvre (Ergon) mais une activité (Energia).» (Meschonnic, 1985, p. 190). Dans l'activité langagière ainsi reconnue comme discours ayant un lieu d'énonciation, le sujet ne peut que constater la perte de toute identité posée antérieurement à la langue. De maternelle et unique, la langue se transforme en familière et plurivoque, sans qu'il soit réellement possible de dire de quelle familiarité il est question ici. Le sujet se reconnaît dans sa production langagière, mais il lui est difficile d'énoncer ce qu'il reconnaît réellement. Il est alors projeté loin d'une langue-mère, 
connue, et se meut dans une langue qu'il s'approprie sans cesse tout en vivant l'éloignement radical de la langue propre qui serait idéalement donnée par la mère.

Dans cette disparition d'une identité sûre et propre au sujet, s'inscrit en même temps une critique de la notion de mot. C'est encore Humboldt qu'il faut convoquer à venir parler de cette dissolution d'un fétichisme du mot:

Dans la réalité, le discours n'est pas composé de mots qui le précèdent mais ce sont les mots au contraire qui procèdent du tout du discours. (Meschonnic, 1985, p. 191)

Si le mot perd sa primauté et si la langue n'est pas assimilable aux «subdivisions traditionnelles» (lexique, morphologie, syntaxe) qu'on lui a attribuées, le propre de la langue pensée comme production incessante et auto-engendrement pourrait se définir comme la capacité de créer des métaphores, et il faudrait entendre ici le mot métaphore dans son sens étymologique de "porter, conduire au-delà», dans un franchissement des seuils de la langue. Ainsi la métaphore dans une langue pensée comme production, poiesis, prend valeur d'opération interne, de franchissement au-delà des seuils critiques que, usuellement pour signifier et communiquer, la langue aplanit ou annule sans les marquer. (Fédida, 1982, p. 37). La métaphore comme métaphore de l'activité d'une langue, d'une poétique, travaille l'intérieur de tout langage et le pose comme langage. Elle définit ce qui lui appartient mais le conduit au-delà de lui-même vers ses limites. La métaphore deviendrait la limite interne et externe du langage. Là où il se donne et là où il se perd.

Le langage qui n'est plus donné d'avance par une langue maternelle absolument stable et présente devient familier au moment même où il fait l'épreuve de l'étranger, de la perte possible de sa familiarité:

Le site de l'étranger à l'intérieur de la langue (...) est le lieu de l'engendrement de la métaphore, laquelle effectue à la fois une traduction et un transfert (...). Une telle traduction suppose bien que celui qui parle sa langue propre se tienne intérieurement à elle en étranger, se laisse ainsi surprendre par la venue de la langue à lui. (Fédida, 1982, pp. 37-38)

Ce que Fédida dit ici de la langue parlée, je tiendrai à le généraliser à toute la langue pensée comme poétique. La langue a besoin d'aller vers sa limite, cet étranger qu'elle porte en elle, pour exister comme langue non plus maternelle dans le sens courant du terme, mais familière et à la fois étrangère. Ce travail que la métaphore effectue à l'intérieur d'une seule et même langue n'est pas toujours apparent à l'intérieur même de la métaphore et par conséquent de la langue. Ce transfert, ce passage inscrit d'un mot à l'autre, ne peut être révélé que dans la traduction interlinguistique comme la nomme 
Jakobson. En effet, par la traduction en une autre langue, la langue découvre ses limites et ses propriétés. Par la traduction, la langue découvre qu'elle est étrangère à elle-même et que la connaissance du sens d'un mot repose toujours sur une traduction possible ou sur une métaphore potentielle. Comme le dit Antoine Berman:

En fait on présuppose toujours que celui qui peut lire l'œuvre dans la langue d'origine est mieux placé pour la goûter et la connaître que celui qui doit se contenter d'une traduction. Celle-ci serait à l'original ce qu'une photo de femme est à une femme réelle. Mais les deux lecteurs ont affaire à un texte étranger, qui leur reste toujours étranger traduit ou non. Cette étrangeté est irréductible. (Berman, 1984, p. 249)

Entre la langue familière et la langue étrangère se met en place un système qui permet à la langue familière de s'inscrire comme impropre et étrangère au sujet parlant. La traduction permet à la langue un véritable dévoilement de ses propres capacités ou impropriétés. La langue familière n'est alors propre que de cette impropriété fondamentale à l'œuvre en elle. En ce sens l'idée d'un original ou d'une écriture pure, sans idée d'une traduction, est un mythe.

C'est la description et la déconstruction de ce mythe d'une préséance de l'original sur la traduction qui est à l'œuvre chez un Benjamin ou chez un Gæthe. Pour Benjamin la traduction permet à l'original d'approcher ce que celui-ci n'a pas réussi à dire. En ce sens la traduction ne saurait être qu'un mouvement infini vers un idéal du langage jamais atteint dans les langues, mais pressenti dans le lien qui les unit les unes aux autres. Pour Gothe la traduction

ne consiste pas à médiatiser des œuvres étrangères pour les seuls lecteurs qui ignorent la langue de celles-ci. Non: la traduction est une expérience qui concerne aussi bien les traduits que les traduisants.

L'ceuvre a besoin d'être traduite, de resurgir, toute juvénile, dans le miroir d'une langue étrangère, pour pouvoir offrir aux lecteurs de sa langue maternelle son visage de merveille, c'est-à-dire son visage d'œuvre tout court. (Berman, 1984, p. 108)

Ici, comme chez beaucoup de penseurs de la langue, il ne peut exister l'idée d'une langue propre ou d'un original sans la mettre en rapport aussitôt avec une théorie de la traduction. Le passage par l'étranger est constitutif d'une langue ou d'un texte et ceux-ci sont fondés dans la possibilité d'être traduits. Écrire, parler, ce serait, par l'étranger, faire signe à un «langage pur» (Benjamin, 1971, p. 266), harmonie de tous ces modes de visée que sont les langues et qui ne peut se dire que dans une langue propre. On voit alors que la langue est prise entre la production d'un original maternel, inaltérable, et un mouvement infini vers un idéal lui aussi inaltérable mais au-delà de la langue maternelle. Dans les deux cas la langue rêve d'une pureté à l'épreuve de tout ce qui pourrait faire faire à la langue un retour sur elle-même et sur l'arbitraire du signe. La langue maternelle pure qui 
fonde un texte original et éternel est arbitraire, soit, mais totalement naturelle à celui qui la parle. La langue qui fait l'épreuve de l'étranger résorbe l'arbitraire du signe dans un mouvement infini de traduction qui ferait apparaître en dernière instance un langage pur où l'arbitraire du signe serait effacé. La pensée de la langue et de la littérature se meut entre deux impératifs catégoriquement inséparables: «Tout doit se traduire» et «L'intraduisible doit être le propre de la langue ou de l'œuvre originale». Berman relèvera en ce sens ces mots de Humboldt:

Si la traduction doit apporter à la langue et à l'esprit de la nation ce qu'ils ne possèdent pas ou possèdent différemment, la première exigence est celle de la fidélité. Cette fidélité doit être dirigée sur le véritable caractère d'original et non [...] sur ce qu'il y a d'accidentel en lui, de même, d'une façon générale, toute bonne traduction doit naître d'un amour simple et sans prétention de l'original. [...] À ce point de vue est nécessairement lié le fait que la traduction porte en elle un certain coloris d'étrangeté, mais les limites à partir desquelles cela devient une faute $[\ldots]$ sont ici très faciles à tracer. Aussi longtemps que l'on sent l'étranger, mais non l'étrangeté, la traduction a atteint ses buts suprêmes, mais là où apparaît l'étrangeté comme telle, obscurcissant peut-être l'étranger, le traducteur trahit qu'il n'est pas à la hauteur de son original. Le sentiment du lecteur non prévenu ne manquera pas la ligne de partage. (Berman, 1984, p. 246)

Pour Humboldt la traduction, si elle met en scène un amour, voire une révélation de l'original, doit s'en tenir à une fidélité à celui-ci. $\mathrm{Au}$ lecteur non prévenu, comme l'expliquera Berman, de décider entre l'étrangeté et l'étranger. "Mais un lecteur non prévenu, qu'est-ce que c'est?» (Berman, 1984, p. 247). La traduction se meut entre un désir d'allégeance totale à l'original et l'impossibilité de définir les critères d'une telle fidélité, car ces critères n'existeraient que dans une langue au-delà de toutes, une langue qui pourrait juger du passage d'une langue à l'autre. C'est sur cette folie de l'acte de traduire que je tenterai de m'interroger. Comment tenir le paradoxe de la traduction qui veut qu'une langue soit propre et intraduisible et qui pourtant présuppose un langage où le passage d'une langue à l'autre trouverait un fondement et une loi?

En fait, la langue place la littérature devant la question de l'arbitraire du signe, et cela bien avant qu'un certain Saussure vienne se prononcer sur ce problème. La littérature a besoin de la pensée de la traduction possible pour faire de l'original une véritable cuvre première. Dès qu'un auteur ne voit plus la langue comme donnée par la mère qui assurerait le sens des mots et le lien de ceux-ci aux choses, la traduction apparait. La langue, ayant perdu toutes ses assises naturelles, veut et doit faire l'épreuve de l'étranger pour fonder en elle le non-arbitraire du langage en général. Par la possibilité d'être traduite, l'œuvre résiste à la traduction et se révèle comme intraduisible et première. De même, le processus de la métaphore voudrait nous 
indiquer que certains mots ne fonctionneraient jamais qu'au sens propre. C'est précisément à partir de ces mots que la langue réussirait à construire ses métaphores. Or, comme l'a montré Derrida dans le Retrait de la métaphore, la phrase de Heidegger «le langage est la maison de l'être» ne nous dit pas ce qu'est le langage à partir du sens propre du mot «maison» si l'on ne sait d'avance ce qu' "être» et «langage" veulent dire au sens figuré. Si «maison» explique «être" et «langage», «être» et «langage» redéfinissent le mot «maison» et lui donnent sa signification. On voit par l'analyse que Derrida fait de la langue que celle-ci ne peut que mythiquement conserver en elle des mots qui seraient d'avance pleins et intouchables. La métaphore fonctionne sur la fiction de mots dont tous connaîtraient le sens et qui ne seraient pas propres à être définis. Des mots formeraient le noyau plein et irréductible de la langue. La traduction, de même, révélerait à la langue son noyau intraduisible, inaltérable, son originalité, alors que pour dire un mot il faut déjà que je sois en mesure de le perdre, et de le traduire pour mieux le définir.

\section{Le noyau pur du langage, l'exemple de Mallarmé}

Quand elle se donne pour fondatrice d'un nouveau langage ou encore lorsqu'elle se pose comme processus incessant et instituant de la langue, la littérature louche du côté de l'étranger en elle, de sa traduction et de sa métaphorisation. On s'aperçoit que nombre d'écrivains ont eu recours à une théorie de la traduction et de la langue pour fonder une langue non arbitraire dont le sens échapperait précisément à ce qui peut être altéré, contaminé et détruit par la traduction. Il s'agit de voir sur quelles bases théoriques une langue poétique individuelle ou nationale se construit et sur quels fondements l'émergence de toute pensée littéraire se bâtit. Sur quel mythe se fonde une langue? Comment celui qui prend la plume, pour transformer et légitimer une nouvelle langue, se pose, de façon détournée ou non, la question de l'arbitraire du signe et de la traduction? Sur quoi se bâtit une langue lorsque ce n'est plus sur sa naturalité donnée en partage à tous les sujets parlants?

Avec l'exemple de Mallarmé et de ce que certains considèrent comme un écrit mineur, les Mots anglais, il est possible d'examiner comment une langue poétique se donne une légitimité et travaille l'arbitraire du langage. Dans les Mots anglais, Mallarmé donne la clé d'un décodage possible de l'anglais. En effet, pour chaque mot il invente une filiation de mots homonymiques. Ainsi, lorsqu'un mot anglais s'énonce, il n'est plus possible de l'entendre seul, mais il faut écouter avec lui tout le réseau de mots qui le doublent. La langue constitue un réseau de mots associés qui commandent le sens de la langue et qui défient tout arbitraire; la langue devient un tissu inextricable et plein de ramifications. Chaque mot en appelle d'autres, et l'arbitraire du rapport signifiant/signifié est occulté, enfoui dans la 
chaîne des mots qui se ressemblent; par ailleurs, cette chaîne ininterrompue permet de développer une théorie sur les premières lettres des. mots. En effet, à partir d'une chaîne de mots de sonorités proches, il est possible pour Mallarmé de déduire que le sens profond de ces mots réside dans leur «attaque», dans leur première syllabe ou leur première lettre. Cette attaque recèle la vérité naturelle du langage et: ne serait absolument pas arbitraire mais justifiée par le rapport des mots et des choses. Par exemple à la lettre $\mathrm{C}$ on peut lire:

Les mots en $\mathrm{C}$, consonne à l'attaque prompte et décisive, se montrent en grand nombre, recevant de cette lettre initiale la signification d'actes vifs comme éteindre, fendre, grimper. (Mallarmé, 1945, p. 940)

Mallarmé dans les Mots anglais donne la clé de l'imbrication des mots et de leur sens dernier. Bien sûr, cette clé est donnée pour les mots anglais et non pour les mots français, mais beaucoup de commentateurs s'en servent néanmoins en français pour traduire et commenter la langue mallarméenne. C'est le cas de Greer Cohn qui fait un véritable travail de traduction de la langue mallarméenne en français d'où le mystère mallarméen aurait disparu. En cela Greer Cohn suit le chemin ouvert par Mallarmé. Celui-ci, en nous donnant les lois et les clés de l'anglais, nous envoie tout au moins sur le chemin du décryptage de tout mot, de toute langue.

Or les Mots anglais présente la langue anglaise comme le lieu de conservation d'un français pur et archaïque que l'histoire du français n'a pas su garder intact. À l'intérieur de l'anglais vivrait la pureté du français, mais une pureté que le français ne saurait approcher sans la faire dégénérer dans un processus historique. Mallarmé écrira en ce sens: «Mille motifs de savoir l'anglais, mais il n'en est point de plus cher que d'y reconnaître les mots français de jadis.» (Mallarmé, 1945, p. 998) Au poète incombe la tâche de "retremper» le mot français à sa source anglaise. L'écriture pour Mallarmé serait une traduction du français vers son origine vivant en anglais. Retrouver la virginité des mots, leur pureté telle qu'elle n'a jamais pu vraiment exister en français, telle serait la tâche de celui qui se donne pour poète. Le français doit retourner vers son origine lovée dans l'anglais et se voit ainsi pris dans un réseau dont il ne peut plus s'extirper. En effet, il vient se mêler à la grande chaîne des mots aux sonorités semblables. Chaque mot s'inscrit, par l'anglais, dans une suite de mots plus ou moins homonymes. Ainsi le mot français trouve une signification dans le lien qui l'unit aux mots anglais. Par cette opération que la langue doit effectuer vers l'autre langue, il nous est dit que sous le texte poétique se cache tout un réseau sous-jacent que la poésie a mis au jour.

Dans les Mots anglais, Mallarmé donnerait la clé de la lecture de ses textes et, du même coup, tenterait de déplacer la question de l'arbitraire du signe en français vers l'anglais et sa continuité homophonique. 
$\mathrm{Si}$, pour Mallarmé, les Mots anglais nous permet de voir ce qu'il en est d'une traduction du français vers un français plus originaire et anglais, il faut tenter de voir que les commentateurs de Mallarmé ont toujours perçu celui-ci comme un écrivain français qui ferait subir à la langue une altération. Le français mallarméen deviendrait un français hermétique où l'étrangeté et l'étranger seraient à l'œuvre. Ainsi Jean Scherer dans l'Expression littéraire dans l'œuvre de Mallarmé cherche à voir quelle fut l'influence des langues étrangères (latin, anglais) sur la langue mallarméenne. Émilie Noulet dans l'CEuvre poétique de Stéphane Mallarmé nous dit que si Mallarmé est obscur et opaque en français, c'est bien à cause de la contamination de la langue française par l'anglais. En fait, il faut bien le dire, Mallarmé est vu par ses critiques comme le plus français des poètes, celui qui a su mener la langue vers sa pureté et sa quintessence, et en même temps, comme l'étranger, celui qui a parasité la langue avec une certaine étrangeté. Or, pour répondre à Émilie Noulet qui voudrait débarrasser la langue mallarméenne de ses influences étrangères, on peut se demander si Mallarmé ne serait pas moins français s'il n'avait pas pensé sa langue comme une traduction vers son noyau pur abrité dans l'anglais. Lorsqu'Émilie Noulet se permet de traduire des poèmes de Mallarmé en français sous prétexte que le texte de Mallarmé est hermétique, on est en droit de croire que Mallarmé fonctionne pour ses commentateurs comme un texte contradictoire où il est impossible de départager l'élément natal, maternel, français, de l'élément étranger, car c'est précisément ce qui est étranger qui fait des poèmes mallarméens le lieu du français le plus pur. Ce qui se joue ici est la nécessité paradoxale de voir le français mallarméen comme le plus français des français tout en constatant une influence étrangère qui brouille les textes de Mallarmé en même temps qu'elle scelle ceux-ci comme authentiquement français.

La langue de Mallarmé, en ces conditions, comporte des mots et des sons étrangers au français courant dans leur arrangement. À première vue, ces mots peuvent apparaitre comme partie intégrante de la langue française, mais ils forment dans la langue une poche étrangère que l'on pourrait éventuellement traduire en français courant. Les mots de Mallarmé forment une enclave dans le français, une enclave étrangère qui rend compte de l'enchevêtrement de toute la langue, enclave française à l'abri du français. On peut alors considérer la langue de Mallarmé comme un charabia non français ou encore penser que, chez Mallarmé, le français ne règne pas totalement sur lui-même, qu'il existe en lui et dans ses énoncés, dans la matière même de ses mots, un réseau qui lui échappe, qui est en lui, mais étranger à lui. Ce réseau ferait signe vers une langue idéale qui serait l'étranger du français à l'intérieur du français. Par la traduction que certains commentateurs opèrent du français mallarméen vers le français courant, l'existence de cette enclave est en quelque sorte affirmée. Le 
texte de Mạllarmé est doublé par une étrangeté à la fois française et étrangère. En fait, pour comprendre l'œuvre de Mallarmé, il faut dans un premier temps reconnaître un effet de brouillage et de parasitage du français, puis, dans un deuxième temps, chercher à voir la distance qui s'installerait entre le texte de Mallarmé et la langue française "courante", si une telle langue existe. Cet écart entre les deux langues serait le mythe constitutif de la lecture de l'œuvre de Mallarmé. Sous le texte réside un autre texte, et si je ne suis pas à même de pouvoir faire cette lecture double du texte, je passe à côté de l'œuvre en la rejetant dans le non-sens.

Mallarmé a donc su créer une grille de lecture de son œuvre en proposant au lecteur un décryptage du sens dans les Mots anglais. À son instar, les commentateurs de Mallarmé ne cessent de livrer, non pas des interprétations, mais d'abord et avant tout des traductions de l'œuvre qui ne se laisse pas appréhender par un simple lecteur de langue française. Ainsi est créée la fiction d'une langue mallarméenne qui, tout en étant française, se tient dans les limites du français, là où la langue peut devenir étrangère. La langue de Mallarmé est une langue prête aux évanouissements, une langue qui se pose en s'effaçant, une langue vacillante du désir de s'instituer pure, tout en étant perpétuellement obligée de nommer l'étranger qu'elle voudrait exclure. La langue française de Mallarmé nomme les endroits où elle est à la limite, aux bords d'elle-même. On ne peut ici s'empêcher de faire appel à Abdelkebir Khatibi qui parle de la langue française confrontée ainsi à son étrangeté: "Assumer la langue française, oui pour y nommer cette faille et cette jouissance de l'étranger qui doit continuellement travailler à la marge, c'est-à-dire pour son seul compte, solitairement". (1983, p. 179) C'est cette faille que Mallarmé appelle à être, mais cette faille dans les Mots anglais n'est pas une absence, un silence, un blanc; elle fait partie de l'ordre de la langue et s'énonce en français dans une autre langue. Cette étrangeté, lieu de la pureté de la langue, peut être entendue dans la langue de Mallarmé. On pourrait écrire ce que Réda Bensmaïa a dit de l'œuvre de Khatibi:

On ne peut éviter d'entendre un grincement, un «bruit», qui sans cesse vient en quelque sorte court-circuiter le mot français, hanter le lexique et faire vaciller la syntaxe de la langue française selon un rythme et une scansion dont la raison demeure indécidable: quelque chose bouge et se déplace constamment et vient brouiller la lecture du texte sans qu'on puisse lui assigner un lieu (géographique, rhétorique). «Un mouvement de déport» (R. Barthes) qui vient perturber la lecture et la rendre difficile d'accès: à tout moment on a le sentiment d'avoir changé de langue, d'être passé d'une langue à une autre, sans qu'il soit possible de dire en quoi ou comment (par quel artifice ou magie). (Bensmaïa, 1987, pp. 141-142)

Or, Mallarmé, par les Mots anglais, tenterait de nous dire justement par quels artifices sa langue est déportée vers sa pureté 
étrangère. Les mots anglais apparaissent comme la clé de l'hermétisme mallarméen. Mais peut-on s'arrêter là et dire que Mallarmé a tout encodé puis tout décodé pour le lecteur, et que les Mots anglais sont la possibilité d'une herméneutique de l'hermétisme? Si Mallarmé crée une langue cachée, lovée dans le français, s'il fait une langue pure pour laquelle il invente des conditions de traduction vers une langue courante, s'il nous pousse à rétablir un texte non plus étranger, mais familier, on est en droit de s'interroger sur la stratégie qu'il met en œuvre. Sur quoi Mallarmé garderait-il silence? Une méfiance s'installe et l'on se demande quels étaient les motifs qui conduisaient Mallarmé à faire don du secret, à dire dans un premier temps qu'il y a un secret, quelque chose à déchiffrer, puis dans un deuxième temps à livrer le mode d'emploi du déchiffrement. On peut alors avancer que Mallarmé est indécryptable en ce sens que le déchiffrement (le dévoilement de l'existence du secret de la langue et du secret lui-même) est nécessaire pour maintenir le secret dans le secret. Mallarmé donne une clé qui ouvre et n'ouvre pas. Tout se passe comme si le désir de Mallarmé de dire le mot pur à l'abri du français "courant» fait jouer les mots avec les sons. Les mots font un détour vers d'autres mots pour dérouter le lecteur et rendre le texte illisible. Puis le texte de Mallarmé peut se retraduire, car Mallarmé en a donné la clé. L'anglais ou la langue étrangère vers laquelle va le français "courant» sert donc d'une part au poète à écrire, à traduire, à énoncer une langue pure qui ne peut s'énoncer que par traduction, déplacement homonymique, car en français la langue pure doit se cacher si elle ne veut pas être dégénérée, et d'autre part l'anglais sert à décrypter, à retraduire le mot vers le français «courant», à encrypter encore, car on ne saura jamais quel aurait été le texte que Mallarmé aurait traduit. L'original d'un français «courant» est à jamais perdu, il n'existe qu'hypothétiquement dans les différentes traductions. Dans ce mouvement infini où il est impossible de savoir ce qui s'encrypte et se décrypte dans l'écriture et la lecture, dans la traduction perpétuelle, tout se traduit et pourtant tout se fige. En effet, si la fiction qu'est la pureté dans l'anglais ou dans le français comme partie étrangère au français permet à Mallarmé de faire croire qu'il traduit et de souffler aux commentateurs de multiplier les interprétations, les traductions, les décodages, les décryptages des œuvres en vers et en prose, et si cette fiction autorise Mallarmé à nous donner pour vrai que ses œuvres sont des traductions du français à l'abri du français, si, en fait, la fiction mallarméenne nous donne à croire qu'elle permet toutes les traductions, toutes les écritures possibles, il n'en reste pas moins que les mots de Mallarmé manquent de titre pour dire que c'est au prix d'une traduction qu'ils sont parlants. Les mots que Mallarmé écrit ne peuvent pas dire qu'ils sont traduits et qu'ils énoncent, dans le secret mal gardé, l'impropriété insaisissable. En effet, si chaque nouveau sens que peut prendre un mot rappelle l'insuffisance et la dégénérescence du français, ce sens n'en éloigne 
pas moins le mot nouveau, crypté, de la possibilité de reconnaître le mot duquel il a été traduit. Lorsque Mallarmé écrit, ses mots peuvent tout dire sauf le fait qu'ils ont été traduits et d'où ils ont été traduits. Mallarmé doit nous dire dans ses écrits théoriques, dans les Mots anglais, que la tâche du lecteur est de déchiffrer le texte mallarméen et que ce texte est encodé, qu'il est du français à l'abri du français. La fondation de l'écriture pour Mallarmé ne peut se faire sans le dévoilement du travail de traduction qu'opère le poète, sans une recherche parallèle qui livre le lieu d'un secret. Mallarmé fait donc, corrélativement à ses écrits poétiques, un récit fondateur, les Mots anglais, un récit de la traduction du français vers la langue mallarméenne pure gisant à l'intérieur du français. Mallarmé ne peut dire, quand il écrit avec ses mots, que ses mots sont dans une crypte intérieure au francais, il ne peut le dire que dans la science à côté, dans ses travaux "sans importance dont il ne sied point de parler», mais qui néanmoins nous indiquent que la langue mallarméenne a effectué une traduction. Mallarmé aurait donc besoin de construire le spectre de la langue française "courante" que ses mots ne peuvent faire vraiment apparaître. Pour Mallarmé il y aurait donc une langue française normale pour pouvoir penser une langue pure. De fait, il ne s'agit pas tant pour le poète d'inventer une langue pure, étrangère au français, que de garantir l'existence d'un original en français "courant" des textes mallarméens. La crypte, la langue pure à partir du français protège et veille bien sûr une langue pure, mais propose en même temps l'instauration d'une langue normative, courante. Mallarmé écrit de telle sorte que, lorsqu'on le lit, on puisse nier son appartenance au français, mais aussi de telle sorte que, lorsqu'on accepte qu'il écrit en français, se dessine en nous l'idée du français normatif et l'idée d'une langue pure et non arbitraire. Les écrits scientifiques de Mallarmé, les Mots anglais, sont là pour témoigner de cette structure qui ne peut d'ailleurs se dire dans l'œuvre, car le propre d'une crypte est d'être tue et ignorée de ce en quoi elle est incorporée. La fondation de cette structure se fait donc à l'extérieur de la structure même. Ce qui est préservé alors, ce qui est vraiment gardé intact, n'est plus le "rêve dans sa nudité», la pureté d'une langue, puisqu'elle est dévoilée, mais bien l'original hypothétique et dégénéré à partir duquel s'élaborerait et se traduirait l'œuvre de Mallarmé. On aura beau chercher de quels mots la crypte protège le langage, sur quelle catastrophe ou dégénérescence la crypte a été fondée, on ne pourra trouver que le silence.

Les commentateurs de Mallarmé peuvent redresser le texte de celui-ci, le traduire, le déchiffrer, le remettre en français, ils ne sauront jamais à quelle langue française Mallarmé échappait, devant quels pièges du langage il fuyait. Si Mallarmé, comme il le dit lui-même, a «perdu le sens des paroles familières», il n'en reste pas moins que 
ces paroles familières sont là, fantômes de ce qui n'a pas eu lieu, de la langue familière courante, fantômes de ce qui ne peut se dire que dans la langue pure, à l'abri de la dégénérescence. Curieusement le secret de Mallarmé serait, par un effet de retour, cette langue normative. L'original de la langue familière, dégénérée et dégénérante, s'établit en blanc, dans le silence, et c'est cette langue que Mallarmé garde intacte, tue. S'il triture la langue maternelle en faisant d'elle une langue étrangère, c'est pour prévenir en quelque sorte l'altération naturelle de la langue française. C'est cette destruction que Mallarmé ne peut nommer et pour laquelle il crée des tombeaux de langues intactes, car la désagrégation de la langue doit lui rester interdite. La langue courante, déchiquetée, mise à nu et sans cesse se violant et violant son origine, ne doit pas être approchée par Mallarmé. La crypte, la langue pure commémore l'éloignement radical d'une langue maternelle impure et dégénérée, l'éloignement de ce qui n'a pas de nom, de ce qui est langue décomposée, langue du hasard et langue auto-destructrice.

\section{La crypte de la langue et sa nécessaire traduction}

Avec Mallarmé on voit que la langue peut être perçue comme un système qui s'institue dans la méconnaissance de ses fondations et de son énonciation qui ne cessent pourtant de travailler ce système. Par ailleurs, la langue se fonde dans la tentative de créer, à travers le processus langagier, le mythe d'un secret conservé à l'intérieur de la langue mais que celle-ci ne livrerait jamais. La langue, ainsi, se poserait comme langue propre à un individu, à un poète ou à une nation, lorsqu'elle pourrait contempler en elle l'idée d'un noyau, d'un tombeau de mots purs que rien ne saurait dire. Un texte et une langue seraient alors la rencontre entre une histoire de la langue inscrite de façon cachée dans celle-ci et le secret de la langue auquel aucune interprétation ne pourrait accéder. Le mythe caché d'une inaltérabilité de la langue fonctionnerait comme constitutif de la fondation de la langue et de l'écriture. Et toutes les couches discursives que l'écrivain mettrait en place pour les donner à lire et à décoder au lecteur ne seraient là que pour empêcher d'accéder au sous-sol fondamental de la langue, là où gît sa pureté, pure de par son inaccessibilité. Interpréter l'écriture, traduire la langue, ce serait d'abord dévoiler les diverses instances de tout discours, en même temps enterrer à jamais le sens premier ou dernier qui aurait été l'original de la langue courante à partir duquel a été formée la langue pure. En ce sens, l'idée d'une interprétation infinie des textes, d'une retraduction de ceux-ci en langue normative, irait dans le sens d'une résistance de l'œuvre à toute reproduction que la reproduction mettrait en évidence. Claude Lévesque nous guide lorsqu'il écrit: 
[...] le commentaire en cherchant l'irréductible originalité de l'œuvre du côté du contenu nécessairement la manque en l'essentialisant. (Lévesque, 1978 , p. 159)

Ainsi à travers le langage se dessinerait le mythe d'une origine pure que l'écriture commémorerait en l'occultant. Tout commentaire recréerait la fiction de cette origine impossible et pourtant se donnant à retrouver. Beaucoup d'écrivains produisent dans leur œuvre cet effet pervers qui consiste à vouloir commenter leurs écrits. Mallarmé, avec les Mots anglais, en est le meilleur exemple, mais l'on peut songer aussi à Heidegger qui justifie sa pensée philosophique grâce à une élaboration sur les liens nécessaires entre le grec et l'allemand. Le grec est pour Heidegger l'endroit où l'allemand pourra se fonder et se régénérer et par là proposer un projet philosophique nouveau. On peut d'ailleurs se demander si ce n'est le lot de tout texte que d'encoder et de donner à lire les conditions de possibilité de ses interprétations futures. De là, l'idée d'une crypte originaire de la langue où la langue serait intraduisible, inatteignable, pure et jamais contaminée par celui qui la parle: elle est construite dans l'espace même de la langue et ne peut célébrer aucune antériorité originelle. Pourtant, à travers cette fiction d'une résistance de la langue à elle-même, la langue est déplacée vers le lieu de son absence, de son non-sens et de sa disparition. Cette crypte mythique de la langue pure, il ne faut pas y toucher, ne pas l'ouvrir sous peine de perdre la langue. Elle renferme le vide de tout langage.

Le langage ne va pas jusqu'au bout de sa possibilité de négation, allant jusqu'à l'impossible, que lorsque, après avoir tout nié autour de lui, il en arrive à se nier lui-même comme positivité. Sans cesse, le langage cherche à se projeter vers le non-langage comme vers sa mort même, sans pouvoir mourir jamais. Une obsession d'absence de langage le porte vers cet horizon mortel où il se décide en vain à disparaître. Le paradoxe du langage: dire dans le langage ce qui est avant le langage, alors qu'il me faut l'exclure pour parler et pour le dire. (Lévesque, 1978, p. 114)

À partir de l'invention d'un lieu pur dans la langue se trace l'idée d'une origine inventée nécessaire à l'élaboration d'une théorie linguistique, d'une langue ou de toute fondation d'un discours. La crypte commémorerait en ce cas l'origine qui n'a pas eu lieu mais qu'il est nécessaire de créer pour faire fonctionner la langue, mais en même temps elle célèbre le vide de l'origine, là où la crypte dit que ce qu'elle contient n'est que son érection. Il faut alors imaginer la crypte comme une enclave à l'intérieur de la langue, enclave où les mots seraient purs et semblables aux choses qu'ils signifient. Or, cette crypte, il ne nous est possible que de l'imaginer. En effet, chaque fois que nous croyons découvrir dans la langue une de ses poches du langage, nous voyons que c'est par ce déchiffrement que se révèle une 
autre crypte plus hermétiquement scellée. La pureté du langage cache chez Mallarmé le secret d'une langue courante qui serait pure, car celée et non dévoilée. Comme le dit Blanchot:

C'est le langage qui serait "cryptique" non seulement dans sa totalité excédée et non théorisable, mais comme recélant des poches, des endroits où les mots se font choses, le dedans dehors, en ce sens indécryptable dans la mesure où le déchiffrement est nécessaire pour maintenir le secret dans le secret. Le code ne suffit plus. La traduction est infinie. (Blanchot, 1980, p. 206)

C'est sur ce secret qui échappe au langage tout en se donnant que s'instituerait la littérature qui, en quelque sorte, construit le mythe d'une langue intouchable que la langue traduirait et dirait sans cesse, sans jamais totalement la donner. Là, à l'intérieur de la crypte, gît, mort vivant, l'illimité du langage, le désir pur de toute contamination. Écrire, parler se feraient à partir du souvenir, de la traduction de ces mots purs enfermés dans la crypte: "Toute parole véritable se souvient de cette séparation par laquelle elle parle.» (Lévesque, 1978, p. 110) En ce sens toute écriture fondatrice serait infinie, car elle n'en aurait jamais fini de traduire cette pureté de la langue, et du même coup elle ne pourrait s'arrêter de dire que crypte il y a. La traduction infinie de cette crypte aménagée à l'intérieur du langage, c'est à Claude Lévesque encore une fois de nous la définir:

Une antériorité préalable à tout sens, une vigilance sans forme et sans nom agissant toujours déjà derrière la signification (et non sur elle) une «affirmation qui toujours se précède pour se dérober au sens de ce qu'elle affirme» (...) la coque du mot se trouve passablement malmenée entre les vagues coupantes du langage: en vérité le mot comme unité -1 l'unité mot - n'y résiste pas. Le mouvement de l'espacement agit sur le mot, le desserre, le dé-limite, l'ouvre à d'autres mots, à d'autres phrases, l'anagrammatise, l'inscrit dans des ensembles instables, non plus des termes (identiques) mais le mouvement qui les écarte d'euxmêmes et les rend étrangers à eux-mêmes. Les pirouettes incessantes du signifiant, le glissement sans fin de "tournures", de métaphores, de métaphores de métaphores qui ne laissent place à aucun propre, à aucune vérité nue, emportent dans leur déferlement toute fixité, toute limite, toute marge calculable, toute frontière infranchissable. (Lévesque, 1978, pp. 119-120)

C'est à partir de cette traduction sans fin de ce qui resterait intact dans la crypte que la langue se fragmente et vit son «essentielle hétérogénéité». La crypte, la pureté de la langue comme espace mythique dévoilerait cette hétérogénéité tout en la gardant sauve.

La langue alors porterait son propre horizon de traduction. Elle inviterait celui qui s'en sert à s'engager en elle sans fin et produirait ses propres conditions d'interprétation qu'il est possible d'interpréter elles aussi à l'infini. Le désir de dévoiler la crypte serait en ce sens le même que celui qui préside à l'envie de parler ou d'écrire. Il faut 
dire avec Benjamin qu'il reste dans toute langue et dans ses produits, en dehors du sens communicable, quelque chose d'incommunicable. Pourtant, si pour Benjamin la traduction n'est qu'une sorte de manière provisoire d'atteindre le noyau de la langue, il n'en reste pas moins que ce noyau ne se donne pas à connaître et que ce provisoire n'est en fait que le mouvement incessant de la création. La traduction n'est plus une particularité de la langue, ou une quelconque opération linguistique, elle est la langue elle-même et la condition de son existence inscrite en elle. Elle affecte ainsi la langue non seulement dans son rapport interlinguistique, mais encore dans ses fondements intralinguistiques. La traduction serait alors le nom par lequel la langue se tait et parle sans pouvoir arrêter le mouvement. Sachant cela, il n'est plus souhaitable de tenter de dire la crypte dans sa pureté, car elle n'existe que dans sa traduction infinie et contient en elle ce qui n'est peut-être qu'un mythe, la totalité de tous les mots du monde. L'écrivain est alors pris dans ce paradoxe décrit par Blanchot (1980, p. 187): «Je voudrais me contenter d'une seule parole, maintenant pure et vive dans son absence si, par elle, je n'avais à porter tout l'infini de tous les langages.» La traduction, elle, permet la restriction du désir de la langue et, en dernière instance, elle permet ainsi la parole, l'écriture et l'avènement de la langue.

La langue maternelle est ventriloque, elle fait dire par la bouche de son double ce qu'elle ne peut émettre sous peine de se contaminer. Elle croit manipuler l'autre et lui faire dire ce qu'elle veut, mais désormais elle ne pourra plus semer ce fantôme qui toujours la doublera. À côté d'elle l'autre veille; cette garde du corps mort de la langue maternelle, qui mime le silence, ne peut être qu'incessante. Entre ces deux langues, une union, une certaine parenté se tracent; elles ne peuvent que se ressembler et l'une d'elles ne cesse de doubler l'autre. Pourtant, dans l'espace qu'elles dessinent se dévoilent les problèmes de l'original et de la copie, de la reproduction et de la filiation des langues et des textes. D'où vient une langue? D'où vient un texte? Nécessairement d'une autre langue, d'un autre texte. Et la langue et le texte inscrivent en eux le lieu de leur filiation tout en se proposant de l'oublier, de se donner comme premiers.

La crypte serait là pour écrire et effacer cette généalogie du texte ou de la langue. Elle se construit comme l'innommable du désir, là où gisent les contradictions et le tout du langage. Mais ce désir ne peut s'énoncer tel quel dans son abjection pure, il a besoin de se restreindre, de se déformer et de se traduire pour venir à la parole. La crypte reste le lieu mythique de cet absolu d'un désir de pureté qui n'existe que dans sa traduction. La crypte n'existe pas, elle n'est que le lieu, l'horizon, vers lequel tous les mots de la langue pointent pour dire leur provenance, qui ne s'énonce jamais qu'en eux. La crypte est alors tout et rien, dans une oscillation perpétuelle. Elle est 
«'indicible en tant que proféré». À partir de là, il faut penser le décryptage, la traduction comme le mouvement même de toute parole, et voir que la littérature se donnerait nécessairement dans ces limites comme critique, déchiffrement d'une parole pure, absolue et inexistante:

Écrire, c'est peut-être non-écrire en récrivant, effacer (en écrivant pardessus) ce qui n'est pas encore écrit et que la réécriture non seulement recouvre, mais restaure obliquement en la recouvrant, en obligeant à penser qu'il y avait une première version (détour) ou pis, un texte d'origine et par là nous engageant dans le processus de l'illusion du déchiffrement infini. (Blanchot, 1973, p. 67)

Tous les mots de la langue semblent convoqués à n'être que la fissure, la défaillance de la langue telle que la crypte la dirait si elle pouvait parler sans les mots, dans le silence ou dans le bruit. De la langue trouée, impropre, à la langue pure, indicible, il existe un passage sans fin. Et la crypte toujours se dérobe. La vérité ne s'écrira encore ici que dans la traduction et la trahison la plus fidèle à ce qui n'a pas eu lieu, la pureté.

Montréal

\section{Références}

ARENDT, Hannah (1980). «Seule demeure la langue maternelle». Esprit $\mathrm{n}^{0} 6$, pp. 19-39.

Benjamin, Walter (1971). «La Tâche du traducteur». Mythe et violence. Paris, Denoël, pp. 261-275.

BENSMAïA, Reda (1987). «Traduire ou «blanchir» la langue». Imaginaire de l'autre. Khatibi et la mémoire littéraire. Paris, L'Harmattan, pp. 113160.

Berman, Antoine (1984). L'Épreuve de l'Étranger. Paris, Gallimard.

Blanchot, Maurice (1973), Le Pas au-delà. Paris, Gallimard.

(1980). L'Écriture du désastre. Paris, Gallimard.

FÉdidA, Pierre (1982). «Le Site de l'étranger», L'Écrit du temps n 2, pp. 35-44.

Humboldt, W. von (1974). Introduction à l'aeuvre sur le Kavi. Paris, Gallimard.

(1960). «Ueber die Aufgabe des Geschichstschreibers». Werke in fünf Bänden. Stuttgart, Cotta'sche Buchhandlung, t. 1, pp. 586-606.

KhatiBI, Abdelkebir (1983). Maghreb pluriel. Paris, Denoël.

LÉvesque, Claude (1978). L'Étrangeté du texte. Essai sur Nietzsche, Freud, Blanchot et Derrida. Paris, U.G.E. 
Mallarmé, Stéphane (1945). Les Mots anglais in CEuvres complètes. Paris, Gallimard, pp. 885-1053.

Meschonnic, Henri (1985). «Poétique d'un texte de philosophie et de ses traductions. Humboldt, sur la tâche de l'écrivain de l'histoire». Les Tours de Babel, Gérard Granel éd., Mauvezin, Trans-Europ-Repress, pp. 181-229. 